miscarriage occurred at three and one-half months, one tumor was entirely absorbed, and the other was unaltered in size. In case $X$. the tumor was sessile and only as large as a goose's egg, but appears not to have been absorbed.

The above experience would seem to warrant the following deductions, which do not make a part of the doctrines hitberto prevalent upon the subject :-

As aids to diagnosis, the following points should have great weight: -

(1) An area of flat percussion beyond the limits of the tumor or tumors.

(2) Unduly rapid growth of a fibroid.

(3) Blueish discolorations of the vaginal entrance. As to treatment:

(4) That intraüterine disinfectant douches should be administered throughout the puerperal period in all cases, even before the supervention of symptoms.

As to prognosis:-

(5) That fibroids are, as a rule, absorbed during involution of the uterus or soon after.

\section{THE INFLUENCE OF OVARIOTOMY ON SURGERY.1}

BY JOHN hOMANS, M.D., OF BOSTON.

THE privilege of addressing an assemblage of men engaged in the same pursuit, and having a sympathetic feeling for each other's endeavors to relieve suffering and lengthen human life, is one which I highly prize.

An audience furnished as this is from the annual meeting of the members of the Massachusetts Medical Society is a sympathetic audience which it is a pleasure as well as an honor to address.

I shall not undertake to enumerate in detail all the operations which have followed the habitual performance of ovariotomy and the familiarity with the peritonaum that this implies. I will, however, later on enumerate many of them and narrate at length some of the most striking.

Many of the gentlemen present to-day will remember that not very long ago the distinction between surgery and medicine, or rather between a surgeon and a physician, was that the former dealt with maladies and diseases on the outside of the body, and the latter with those affecting the internal organs, - the principal exceptions to this rule being in the case of foreign bodies in the bladder or airpassages. At the period referred to there were no special courses except on ophthalmology. Now the manner of teaching the science and practice of medicine and surgery has changed and developed so much, particularly during the last ten years, that one in search may find somewhere a special course on almost any subject connected with medical or surgical knowledge. Among the branches of practical surgery none has made greater strides, or been more fascinating in its performance, than abdominal surgery. Twenty-five or thirty years ago, the interior of the abdominal cavity, except to an occasional ovariotomist, or to a performer of Cæsarean section, was a terra incognita, and not only unknown, but feared and dreaded. The occa-

1 Read before the Massachusetts Medical Society, June 10, 1855, and recommended for publication by the Society. sional, I had almost said every-day, occurrence of stabs in the abdomen letting out the intestines and followed by recovery, taught surgeons nothing in regard to the harmlessness of simple incision of the peritonæum, and each case was treated with dread, and the rapid convalescence was recorded with wonder and reported as extraordinary. Still more severe injuries, caused by stakes or pitchforks penetrating the abdomen, only served to cause the instruments of these wounds to be preserved in museums, and the patients to be exhibited as surgical curiosities. No one saw and acted on the evident truth that a simple incised wound of the abdominal parietes was almost innocuous. This dread of the peritonæum was caused partly by the experience of surgeons in herniotomy delayed too long, or by seeing peritonitis and death follow a wound of the peritonæum made in a vaginal surgical operation; and by the experience of physicians in autopsies following septic peritonitis after childbirth, in which the intestines were found of a deep purple color and glued together by lymph and pus. All these experiences made the surgeon of twentyfive years ago fear to wound the peritonæum, and held him back from opening it voluntarily and exploring its cavity. To look back now on the long list of recorded cases of men who were found in the streets with incised wounds of the abdomen, and whose intestines, covered with dirt, were carefully washed and replaced within the belly and kept there by sewing the wounded walls together, and who almost invariably recovered, makes us wonder that some one did not see that, if this class of wounds was followed by recovery, how much more likely were wounds carefully made by a cleanly surgeon to unite and heal up !

The literature of abdominal surgery is to-day so voluminous that one man can scarcely read all that comes out in the periodicals alone. The operation of abdominal section, or opening the peritoneal cavity by an incision, has been called laparotomy, from the Greek word lapara, which means the soft parts of the body between the ribs and hips, and $\tau 0 ;, y$, an incision.

Such words seem pedantic at first, but laparotomy is more concise than " abdominal incision," and we may as well use it. In the textbooks before 1850 , ovariotomy is only alluded to, and laparotomy, for the purpose of learning what the trouble was inside the belly, was not dreamed of. I never saw ovariotomy done in this city before I did it, and when I studied medicine here it was not mentioned in lectures or in recitations on practical surgery, and its performance was discountenanced and discouraged by the highest surgical authorities in this neighborhood. Dr. Gilman Kimball, of Lowell, however, had been for many years practising ovariotomy in New England, and pursuing his work with great courage and enthusiasm. But by the profession generally the operation was not regarded favorably. Dr. Burnhan, of Lowell, was also operating, and in other parts of the United States Atlee, of Pennsylvania, and Peaslee, of New York, were active ovariotomists. Spencer Wells, of London (now a baronet, Sir Spencer Wells, on acount of his triumphs in ovariotomy), had taken up the subject on his return from the Crimean War, where he had 
served as a surgeon, and between 1858 and 1864 had operated one hundred times with thirty-four deaths. Although this was a high mortality, yet Sir Spencer proved conclusively that the operation was not only justifiable but imperative.

To Mr. Charles Clay, of Manchester, England, credit is also due for reëstablishing the operation, but I think Sir Spencer Wells's plan of showing all his specimens at Society meetings in London, and reporting every one of his cases, caused the revival and establishment of the operation.

I will not trace the history of ovariotomy, from its first performance by McDowell, of Kentucky, in December, 1809, to the present time; but will enumerate some of the operations and triumphs of abdominal surgery which have grown out of the familiarity with the peritonæum brought about by ovariotomy.

The first operation which followed the removal of ovarian tumors was the removal of fibroid tumors of the uterus. This operation is only to be done in exceptional instances, and its success will probably never equal that of ovariotomy, but the splendid results of Dr. Keith, of Edinburgh - thirty-five cures out of thirty-eight operations - show us what can be accomplished in the performance of this very formidable operation. When it was found that wounds of the intestine, made during an ovariotomy, often united when carefully sewn together, the natural inference was made that intentional wounds of the bowel could also be healed, and this inference was acted on and resection and suture of the intestine for the cure of fæcal fistula was successfully done.

'Two cases of artificial anus have been cured by this operation by Dr. Porter at the Massachusetts General Hospital within the last eighteen months. It seems very hard, even now, to believe that the intestine can be pulled out of the abdominal cavity, pared, sewed together, and returned, and the abdominal wound completely closed at once and a cure result. But $I$ have myself seen it three times, and seeing is believing. Who does not remember some dreadful sufferer with an intestinal fistula following a strangulated hernia, dragging on a miserable existence, avoiding and avoided, without control over his offensive fæcal emanations, solid, liquid, or gaseous. Now to-day ovariotomy has made possible an operation by which this sufferer can be made completely well again.

Laparotomy is done for operations likely to be successful, often or seldom, for cases almost sure to recover, and in desperate cases as a last resort, a forlorn hope. By means of it the spleen has been removed, cancers of the stomach and intestines have been cut out, gallstones have been removed from the gallbladder, foreign bodies from the stomach and bowels, calculi from the kidneys, and even cancerous and diseased kidneys have been excised. The pain and discomfort from floating kidneys has been relieved by sewing the kidney to the abdominal parietes and fixing it in place. All of these operations have been successful in numbers of instances. Perhaps one of the most remarkable instances of successful abdominal surgery is the recent case of Dr. Bull, of New York, who opened the abdomen in a case of pistol-shot wound, found seven perforations of the bowel, sewed the holes together, and cured the patient completely. I saw Mr. Thornton last summer, in London, lay open the stomach and remove a mass of hair shaped like a sausage and nine inches long by two thick. After the removal of this mass, the wound in the stomach was very carefully and patiently sewed together, and the woman did not even vomit during her convalescence, which was uninterupted. It may be interesting to say that the mass of hair was the accumulation of nearly twelve years, during which the woman had swallowed what she combed out each night and morning. Mr. Tait, of Birmingham. has shown that great suffering is caused by pus in the Fallopian tubes, and has cured many cases by removal of the tubes. You would be surprised to see how large these tubes, distended with pus, sometimes become. I have seen them of the size of a cow's horn, twisted. and convoluted. Removal of the ovaries for hysteria, for insanity, and for the cure of painful menstruation (Battey's operation) has also been done. The sphere of this last operation should be closely and carefully limited, but in certain cases it is a proper proceeding. Removal of the uterine appendages, to bring about atrophy of uterine growths, is very legitimate and promises well. Laparotomy has been done successfully and unsuccessfully in cases of intestinal obstruction. As the diagnosis of the causes of obstruction becomes more precise, so will its relief by surgery be more certain. Up to the present time the failures, I think, outnumber the successes. Laparotomy is also properly done to ascertain the character of growths within the abdomen when we are ignorant of their nature and uncertain whether we can remove them.

Simple laparotomy, properly done in a healthy subject, has no mortality, or perhaps a very small percentage, and is practically innocuous. All of these advances in abdominal surgery, all of these triumphs and discoveries in an unknown region of the body, have been caused, brought about, and made possible by ovariotomy.

Encouraged by the success of operations in the abdominal cavity, the thorax is beginning to be explored, and the practice of surgery will probably be extended in this direction. I might give an account of the rise and progress of ovariotomy, and lengthen the list of abdominal operations which have followed it, and keep your interest and attention for many hours; but I have only tried to give a sketch of the influence of ovariotomy on modern surgery. A list of all the operations born of ovariotomy, which have been done successfully, would be long and tedious, but I will venture to enumerate a few. Enucleation, per vaginam, of the entire uterus for cancer of the body of the organ. (This procedure is still on trial, but in practised hands its reputation will improve.) Removal of the spleen for cystic disease; removal of the gallbladder; cutting into the gallbladder (cholecystotomy) ; hepatotomy ( $\mathrm{r}$ incision of the liver) for abscess and for hydatids; nephrectomy (or cutting out the kidney) for calculous pyelitis, for cancer, or for tubercle; nephrotomy (cutting into the kidney) for abscess, or for purposes of exploration. Radical operation for the cure of hernia, by sewing together the pillars of the ring and the sides of the sac. 
Laparotomy has also been successfully done for pelvic abscess, for splenic abscess, for acute and chronic peritonitis, for hydatids of the peritonæum, for extraüterine fotation. Supra-vaginal hysterectomy in pregnancy with contracted pelvis (Porro's operation) has been often successful as a substitute for Cæsarean section; hysterectomy for the cure of uterine tumors I have already alluded to. 'That the abdomen could be opened, and an aneurism of an abdominal artery could be successfully treated, would not have entered into the wildest dreams of the most enthuiastic surgeon a score of years ago, and yet this has been accomplished lately by Professor Loreta, of Bologna. He opened the abdomen and found an aneurisin of the superior mesenteric artery; he tried to tie the artery above the aneurismal sac, but found this impossible on account of the adhesions of the sac to the neighboring viscera; by puncturing the sac with a small needle and introducing two yards of fine copper wire he succeeded - in producing coagulation and curing the aneurism.

My own operations now number two hundred and seventy laparotomies, of which two hundred are ovariotomies for the removal of cystic ovaries; several are cases of removal of ovaries and tubes for the cure of threatened or actual insanity; a very remarkable case of removal of a fibroid tumor of the abdominal parietes and peritonæum, and other laparotomies in which the abdomen was opened for various reasons.

I may be pardoned for uarrating at length another very remarkable instance of the curative influence of an abdominal incision. It is a case of tubercular peritonitis. A single girl, twenty-one years old, feeble, pale and emaciated, with a large belly full of fluid, came to St. Margaret's Home a year ago. It was supposed that she had an ovarian cyst. After she was etherized I saw that the fluid was probably ascitic; but I made an antiseptic (spray) exploratory incision to find out the cause of the dropsy. Much ascitic fluid ran out, and many flakes, masses, and layers of lymph. Tubercular deposits were seen scattered over the peritonæum and bowels. In short, the disease was tubercular peritonitis, as was shown from the gross appearances, and from the microscopic ${ }^{2}$ examination of masses of the peritonæum which I cut away. In about two weeks the wound opened spontaneously to allow the ascitic fluid, which had accumulated, to run out. The girl went home at the end of three weeks, and returned to the care of Dr. Tower, of South Weymouth. At the end of four months she had gained considerable flesh, and had a good appetite; occasionally she walked out when the weather was pleasant. All this time the opening in the scar of the abdominal incision had been discharging serum. In February of this year, the catamenia, which had been absent more than a year, reappeared, and have returned regularly since. I learned from Dr. Tower that the wound is now healed, and the general health has improved wonderfully, that she is fat and of good color, and is contemplating matrimony. 'This is, so far as I know, a unique case, and its treatment and cure are direct outgrowths from ovariotomy.

I have given but a brief sketch of what ovariotomy has led up to, of the successes and triumphs which the establishment of the harmlessness of opening the peritonæum has achieved, and I have shown that all these successful operations within the abdominal cavity owe their inception and execution to the knowledge gained by the ovariotomist and furnished by him to the profession.

"We should count time by heart throbs, not by figures on a dial." We should weigh the results and influences of our work, and not simply count and enumerate our operations.

I care but little for a man's statistics unless I know the character of his work. One may cure all his cases, another may have a mortality of twenty per cent. The former mav have an exceptionally favorable series of cases. "You cannot juclge which has really done the most good, simply by the reported statistics.

But these remarks are foreign to the subject of my address. I only mean to say that on this day, the anniversary of our Society, it would be well for us to weigh our deeds and see what we have accomplished in our lives, and I am sure we should find that we have made immense advances in the treatment of morbid states of the organs within the abdominal cavity; and I think you will all agree with me that to the establishment of ovariotomy we owe all our progress in this direction.

\section{THE STUDY OF ANATOMY:}

ITS POSITION IN MEDICAL EDUCATION IN FNGLAND AND IN AMERICA.

BY G. H. MONKS, M.D.

AN American visiting the English medical schools must be struck with the amount of attention everywhere given to the study of anatomy, not only as an abstract science, but also in its more practical relations to clinical work. It may be interesting to examine the system followed there.

The regular lectures on descriptive anatomy are delivered, as elsewhere, by the professors, and present nothing peculiar. The recitations are, however, so far as I know, conducted almost exclusively by the demonstrators. Quain is the textbook preferred.

Little or no dissecting work is done without the help of some good manual of dissections. Ellis's guide is considered the most reliable and exhaustive; but those of Heath and Holden are also good and are considerably used. The demonstrators spend many hours of each day in the dissecting rooms, demonstrating and examining the students on fresh dissections. Material is so searce that all sorts of means are used to preserve it; and six or seven or even more students work upon the same body. The student has access to numbers of carefully made anatomical preparations, which have been so selected as to embrace nearly all the important parts of the body.

These are arranged with the special purpose of allowing the student to examine them and study from them to the best advantage. In most cases each structure is carefully labeled with printed tags. Some preparations, however, have no labels whatever, and are intended for purposes of exami- 